\section{MAXWELL: LA TEORÍA ELECTROMAGNÉTICA DE LA LUZ}

\author{
Joel Gabàs Masip \\ Universitat de Lleida \\ joel.gabas@udl.es
}

Cómo citar este artículo/Citation: Gabàs Masip, J. (2015). "Maxwell: la teoría electromagnética de la luz". Arbor, 191 (775): a265. doi: http://dx.doi.org/10.3989/ arbor.2015.775n5004

Recibido: 11 diciembre 2014. Aceptado: 6 marzo 2015.

RESUMEN: Este artículo pretende explicar en qué contexto se desarrolló la teoría de las ondas electromagnéticas y se identificó la luz como una de ellas. Para ello se hace un análisis de la figura de Maxwell y su tiempo, haciendo especial hincapié en el estado del conocimiento en óptica, electricidad y magnetismo.

PALABRAS CLAVE: Luz; onda; electromagnetismo.

\section{MAXWELL: THE ELECTROMAGNETIC THEORY OF LIGHT}

Copyright: (C) 2015 CSIC. Este es un artículo de acceso abierto distribuido bajo los términos de la licencia Creative Commons Attribution-Non Commercial (by-nc) Spain 3.0.

ABSTRACT: This article aims to explain the context in which the electromagnetic wave theory was developed and light was identified as an electromagnetic wave. With this aim, it explores Maxwell's life and times, focusing in particular on the state of knowledge of optics, electricity and magnetism.

KEYWORDS: Light; wave; electromagnetism. 


\section{GLENLAIR 1861}

"Para finalizar tenemos las ciencias eléctrica y magnética, que tratan de ciertos fenómenos de atracción, calor, luz y acción química, dependiendo de las condiciones de la materia, de las que solo tenemos hasta ahora un conocimiento parcial y provisional. Una inmensa cantidad de hechos ha sido recopilada y éstos han sido reducidos a orden, y expresados como los resultados de un número de leyes experimentales, pero la forma en que estas leyes están por aparecer finalmente como deducidas de principios centrales es hasta ahora incierta. La generación actual no tiene derecho de quejarse por los grandes descubrimientos ya realizados, como si no dejaran espacio para proseguir la empresa. Tan solo han dado a la ciencia una base más amplia, y no debemos solo reducir a orden las regiones ya conquistadas, sino mantener operaciones constantes a una escala continuamente creciente."

(James Clerk Maxwell, poco antes de predecir las ondas electromagnéticas).

Tras ser nombrado miembro de la Royal Society la primavera de 1861, Maxwell fue a pasar los meses vacacionales de verano a su propiedad de Glenlair en el Sudoeste de Escocia. Era una finca rústica de paisaje ondulado. Contaba con una casa situada donde un pequeño arroyo se encontraba con el rio Urr. La iglesia y el mercado más próximos se encontraban a varios kilómetros de distancia. Desde la muerte de su padre, él era el máximo responsable del lugar. Organizaba las tareas en la propiedad y se reunía activamente con otros miembros de la comunidad. Dedicaba también parte de su tiempo a recibir a parientes y amigos, a actividades de ocio como montar a caballo y evidentemente a su trabajo científico.

En esa época, estaba ocupado en la continuación de su artículo "On Physical Lines of Force" publicado en dos partes en marzo y abril de ese mismo año en The London, Edinburg and Dublín Philosophical Magazine and Journal of Science. En él daba cuenta de las fuerzas magnéticas, las corrientes eléctricas y la relación entre ambos fenómenos. A tal efecto, había ideado un modelo constituido por vórtices cuya rotación provocaba un estado tensional equivalente a las líneas de fuerza magnética descritas por Faraday. Para que no hubiera rozamiento entre los vórtices introdujo unas capas intermedias de pequeñas partículas, que si se consideraban de naturaleza eléctrica podían dar cuenta de las corrientes.

Lo que estaba intentando entonces era incorporar las fuerzas electroestáticas a su modelo de vórtices rotatorios y pequeñas partículas intermedias deslizantes. Fue ese verano en Glenlair cuando se inspiró para encontrar la pieza que le faltaba a su rompecabezas. Se trataba de dotar al sistema de elasticidad e identificar las fuerzas electroestáticas con las tensiones provocadas por la elasticidad transversal de los vórtices rotatorios. El desarrollo de esta idea le llevó a la convicción que los fenómenos electromagnéticos se propagaban en forma de ondas transversales. Al ser similares a las ondas lumínicas, intuyó que quizás ambos fenómenos tenían lugar en el mismo medio.

Para comprobar su intuición, tuvo que esperar al comienzo del curso en el King's College de Londres dónde era profesor y tenía sus libros. Cuando llegó en otoño, consultó los datos que necesitaba y realizó varios cálculos. El resultado fue sencillamente concluyente. La velocidad de propagación de los fenómenos electromagnéticos en forma de ondas transversales que le permitía calcular su modelo coincidía con la velocidad de la luz medida con medios ópticos.

Figura 1. James Clerk Maxwell en la época en que predijo la existencia de ondas electromagnéticas

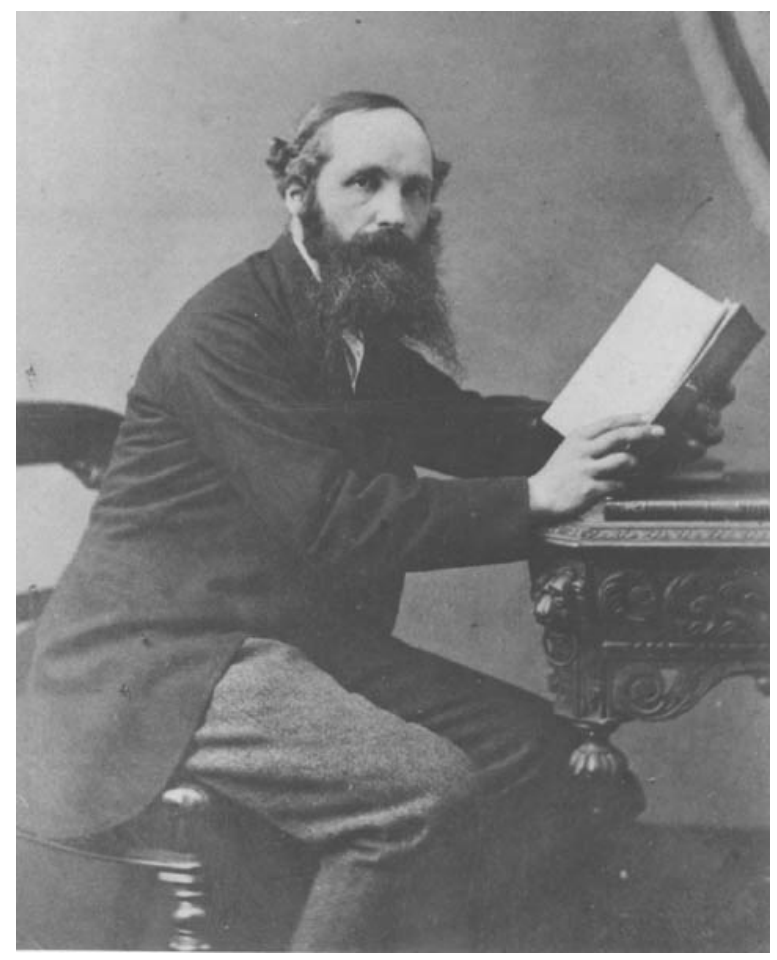

JAMES CLERK MAXWELL

El pequeño James nació en Edimburgo en 1831. Sus padres se habían trasladado allí para el parto, pero enseguida volvieron a su propiedad de Glenlair en el Suroeste de Escocia. Allí pasaría su infancia corre- 
teando por los campos e inspeccionando todo aquello que le llamaba la atención. Era un niño con curiosidad inusual. Pasaba horas intentado seguir los cursos de agua que atravesaban la propiedad así como los circuitos que hacían sonar las campanas para el servicio. Preguntaba constantemente a sus padres sobre todo aquello que caía en sus manos hasta que le contaban todo lo que sabían. Su tía Jane reconocía que era humillante no poder responder a las preguntas de ese niño como "Pero, ¿cómo puedes saber que es azul?".

Su madre, que se ocupaba de su educación, murió cuando James tenía ocho años. Tras algún intento fallido de escolarización con un tutor en casa, a los diez años fue enviado a vivir con su tía Isabella en Edimburgo. Allí estudiaría en la Academia y posteriormente en la Universidad. Durante este periodo realizó sus primeros artículos científicos que fueron leídos en la Edinburgh Royal Society (Maxwell, 1849, 1851, 1853). Sin embargo, no fue considerado apropiado que los leyera él mismo al ser un adolescente.

Tras cumplir los diecinueve años se trasladó a estudiar matemáticas a la Universidad de Cambridge. Allí entró en contacto con los conceptos matemáticos y físicos más avanzados de su tiempo. También tuvo la ocasión de entablar relación con algunos de los autores que los habían desarrollado como George Stokes o WiIliam Thomson (posteriormente Lord Kelvin). Después de graduarse escribió sus primeros artículos científicos importantes (Maxwell, 1855, 1864¹). En el primero exponía los principios de la combinatoria de los tres colores primarios. En el segundo, leído entonces aunque publicado años más tarde, describía mediante ecuaciones diferenciales el estado producido en el medio por los fenómenos electromagnéticos, que Faraday había descrito cualitativamente con sus líneas de fuerza.

En 1856 se presentó y obtuvo la plaza de profesor de Filosofía Natural en el Marischal College de Aberdeen, al noreste de Escocia. Allí entablaría amistad con el director de esa institución, el reverendo Dewar, con cuya hija Katherine Mary contraería matrimonio. Durante su estancia en Aberdeen, ganó el Premio Adams, otorgado por el St. John's College de la Universidad de Cambridge, con un trabajo en que demostraba que los anillos de Saturno estaban compuestos de pequeñas partículas de materia (Maxwell, 1859). También realizó importantes trabajos sobre termodinámica y teoría cinética de los gases (Maxwell, 1860a, 1860b).

En 1860 el Marischal College y el King's College se fusionaron para fundar la Universidad de Aberdeen. No había necesidad de dos profesores de Filosofía
Natural y siendo el más joven perdió su plaza. Afortunadamente al poco tiempo quedó vacante una plaza en el King's College de Londres, a la que se presentó y obtuvo. Los años en el King's College de Londres fueron los más productivos de su carrera. Sus aportaciones más destacadas fueron en electromagnetismo siguiendo la línea de su primer trabajo en Cambridge. Sin duda la más importante fue la predicción de la transmisión de los fenómenos electromagnéticos a través de ondas y la identificación de la luz con una de ellas (Maxwell, 1861a, 1861b, 1862a, 1862b).

Realizó muchas más contribuciones en electromagnetismo como el análisis dimensional o la determinación de constantes. También redactó las ecuaciones diferenciales que describen el campo electromagnético, cuya versión refinada ha llegado a nuestros días como las Ecuaciones de Maxwell (Maxwell, 1865).

Paralelamente prosiguió con sus trabajos en otros campos como la visión del color y la termodinámica, que no fueron menores. Su trabajo sobre la percepción de los colores le había valido ya la medalla Rumford y junto al de los anillos de Saturno la elección como miembro de la Royal Society. En termodinámica fue un pionero en aplicar leyes estadísticas a las partículas para obtener leyes generales de los gases. En ocasión de una charla en la Royal Institution sobre la percepción de los colores a través de los tres primarios ideó un sistema para que los asistentes comprendieran mejor en qué consistía. Sacó fotografías a una misma cinta de cuadros con tres filtros y proyectó las tres fotografías sobre una pantalla. Seguramente se trata de la primera fotografía en color de la historia.

En 1865 dejó sus responsabilidades en Londres y se instaló con su mujer en Glenlair. Así tendría tiempo para encargarse de su propiedad y terminar de escribir su tratado sobre electricidad y magnetismo que publicaría ocho años más tarde (Maxwell, 1873). En estos años de retiro, no dejó sin embargo de escribir artículos, ni de hacer viajes anuales a Cambridge y Londres, incluso hizo uno por Europa.

En 1871 obtuvo el encargo de planificar la construcción del laboratorio Cavendish de la Universidad de Cambridge y convertirse en su primer catedrático de física experimental. En esta legendaria institución se identificaría pocos años después la primera partícula subatómica, el electrón. Desgraciadamente no pudo ver ni el gran éxito del laboratorio Cavendish ni la demostración experimental de su teoría de las ondas electromagnéticas a cargo de Heinrich Hertz. En 1879, como su madre, falleció a la edad de 48 años a causa 
de un cáncer abdominal. Fue enterrado en una iglesia cercana a Glenlair junto a sus padres.

Para aquellos interesados en el personaje, existen varias biografías escritas poco después de su muerte por amigos y conocidos (Campbell y Garnett, 1884; Glazebrook, 1896). Por otro lado, existen ediciones de sus escritos científicos recopilados (Niven, 1890). También existen publicaciones que ofrecen una visión actual sobre su persona y obra (Mahon, 2003). Cuanto a las versiones en español, existen varias traducciones (Crowther, 1945; McDonald, 1966) y libros originales (Ladera y Chela, 1979; Gabàs, 2012).

\section{CONTEXTO HISTÓRICO}

En la primera mitad del siglo XIX Gran Bretaña se encontraba en plena revolución industrial. Se había puesto a la cabeza a nivel mundial en la extracción de carbón, metalurgia y producción de bienes materiales. Su población estaba aumentando de forma exponencial y concentrándose en las ciudades. La burguesía urbana que dominaba los procesos productivos cada vez tenía un papel más importante. Se estaba desarrollando también de forma incipiente una red de transportes terrestres de mercancías y personas gracias al ferrocarril que permitiría una movilidad más rápida y confortable. El dominio de la tecnología de la máquina de vapor aparecía como el motivo de todo aquel progreso y se hacían esfuerzos importantes para comprender los mecanismos últimos de su funcionamiento. Esto supuso un gran empujón para la ciencia al ser percibida como una fuente de riqueza. Íntimamente relacionado con la optimización del rendimiento de las máquinas de vapor se favoreció el estudio del comportamiento de los gases y del calor (teorías atómica, cinética, termodinámica y química). Por otro lado se encontraron las primeras aplicaciones prácticas para la incipiente ciencia electromagnética como la transmisión de información por cable.

En la segunda mitad de siglo XIX se empezó a desarrollar una red telegráfica mundial utilizando la tecnología de los cables submarinos. Se abría así la posibilidad de establecer comunicaciones inmediatas con cualquier parte del mundo que estuviera conectada. Gobiernos, banqueros, comerciantes o agencias de noticias podrían contactar con sus diplomáticos, clientes, proveedores o corresponsales para saber que estaba pasando en la otra punta del mundo en tiempo real. Se podrían cerrar transacciones con personas a miles de kilómetros de distancia. Dado el valor estratégico del control de las telecomunicaciones, era perfectamente comprensible que se hicieran grandes es- fuerzos en el desarrollo de la tecnología del telégrafo y en la comprensión del fenómeno electromagnético. Este fue pues un tema muy presente en el trabajo de Maxwell y su punto de partida para llegar a su teoría electromagnética de la luz. Antes de tratar el fenómeno electromagnético en profundidad, veamos cual era el estado de la óptica.

\section{ÓPTICA}

En la antigua Grecia ya se había especulado sobre la naturaleza de la luz y si se transmitía con una velocidad finita o de forma instantánea. Según la escuela de los atomistas, los objetos podían verse gracias las partículas que emitían. Por otro lado, en la óptica de Euclides ya aparecía una explicación geométrica de la sensación de perspectiva. En el siglo XI, el oculista árabe Alhazen construyó una teoría de la visión recogiendo lo mejor de las anteriores y añadiendo una explicación física más satisfactoria: los rayos de luz, de procedencia exterior al observador, rebotaban en los objetos y eran percibidos por su ojo. A principios del siglo XVII, tras la generalización de las cámaras oscuras en la elaboración de bocetos y pinturas, René Descartes describió el mecanismo por el cual los rayos de luz se proyectan sobre la retina como una imagen invertida. Ese mismo siglo se propuso la teoría ondulatoria de la luz y se produjeron los primeros intentos de medición de su velocidad de propagación (Hoffmann, 1983).

\section{La teoría ondulatoria de la luz}

Uno de los primeros en proponer una teoría ondulatoria de la luz fue el físico y matemático holandés Christian Huygens en el siglo XVII. Se basaba en que cada punto de un frente de ondas lumínicas podía considerarse como una nueva fuente. La teoría ondulatoria de la luz sufrió un impulso definitivo a principios del siglo XIX cuando Thomas Young realizó una serie de experimentos que la apoyaban. En uno de ellos, interponía una tarjeta de $0.2 \mathrm{~mm}$ a un haz de luz procedente de un pequeño agujero en una habitación oscurecida redirigido mediante un espejo. Ligeramente más ancho que la tarjeta, el haz quedaba dividido en dos antes de proyectarse sobre la pared. En esta proyección se podía observar un patrón de bandas claras y oscuras parecido a otros fenómenos ondulatorios. Young propuso también que los distintos colores correspondían a distintas frecuencias de onda y que la visión se articulaba a través de tres únicos tipos de receptores en la retina para sendos colores primarios. 
En las décadas siguientes al experimento de Young, Augustin Fresnel dio rigor matemático a esta teoría considerando que las ondulaciones lumínicas eran transversales y no longitudinales como se había creído hasta entonces. Se basaba en que dos haces de luz polarizada de forma paralela interferían, aunque no así si estaban polarizados con un giro de 90 ․ Por otro lado, las ondas transversales necesitaban un medio de propagación sólido o al menos dotado de cierta rigidez o elasticidad transversal. Así que, se consideraba para las ondas lumínicas un medio etéreo con tales características.

\section{La velocidad de la luz}

Hasta el siglo XVII no se conocen los primeros intentos de medir la velocidad de la luz. Galileo narra un experimento en que se situaba a dos personas tapando una lámpara en montículos situados a aproximadamente un kilómetro de distancia. Una destapaba su lámpara y la otra al verla destapaba la suya y cuando se veía desde el primer montículo se podía medir el tiempo que tardaba la luz en su viaje de ida y vuelta. Concluyó que la velocidad de la luz no podía medirse con ese método ya que, si era finita, era mucho mayor que la velocidad de reacción de los individuos. Sin embargo, sus aportaciones en astronomía posibilitaron la primera aproximación aceptable unos años más tarde.

En la década de 1670, el astrónomo Ole Rømer observó que el periodo de revolución del satélite ío alrededor de Júpiter se alargaba cuando este planeta y la tierra se alejaban y se acortaba cuando se apro- ximaban. Encontró una explicación a este fenómeno utilizando la velocidad de la luz. Cuando se estaban alejando, la distancia que tenía que recorrer la luz iba aumentando y el periodo de ĺo visto desde la tierra parecía más largo. Contrariamente, cuando se estaban aproximando parecía más corto. Si se sumaban todos estos retrasos o avances al aumentar o disminuir la distancia entre ambos planetas, se obtenía que: la luz tardaba 22 minutos en recorrer el diámetro de la órbita terrestre. Con el conocimiento actual de las distancias astronómicas esto equivaldría a unos 210.000 km/s.

Las primeras mediciones precisas realizadas exclusivamente con aparatos terrestres se produjeron en el siglo XIX. En 1849 Hippolyte Fizeau realizó un experimento conceptualmente muy parecido al de Galileo. Consistía en una fuente de luz que emitía un rayo que se reflejaba en un espejo a centenares de metros de distancia. Entre la fuente y el espejo había una rueda dentada rotatoria. Ajustando su velocidad de rotación se podía conseguir que el rayo que de ida había atravesado una ranura impactase de vuelta contra un diente. De esta manera los rayos emitidos por la fuente no serían visibles desde esta. Conociendo las dimensiones, la distancia al espejo y la velocidad de rotación de la rueda que producía la invisibilidad de los rayos, se podía saber cuánto tardaba la luz en hacer el recorrido y su velocidad. El resultado fue de unos $314.000 \mathrm{~km} / \mathrm{s}$. Este método fue perfeccionado por Léon Foucault reemplazando el engranaje por un espejo rotatorio obteniendo un resultado de $298.000 \mathrm{~km} / \mathrm{s}$ (menos de $1 \%$ de error).

Figura 2. Esquema del experimento de la doble rendija de Young

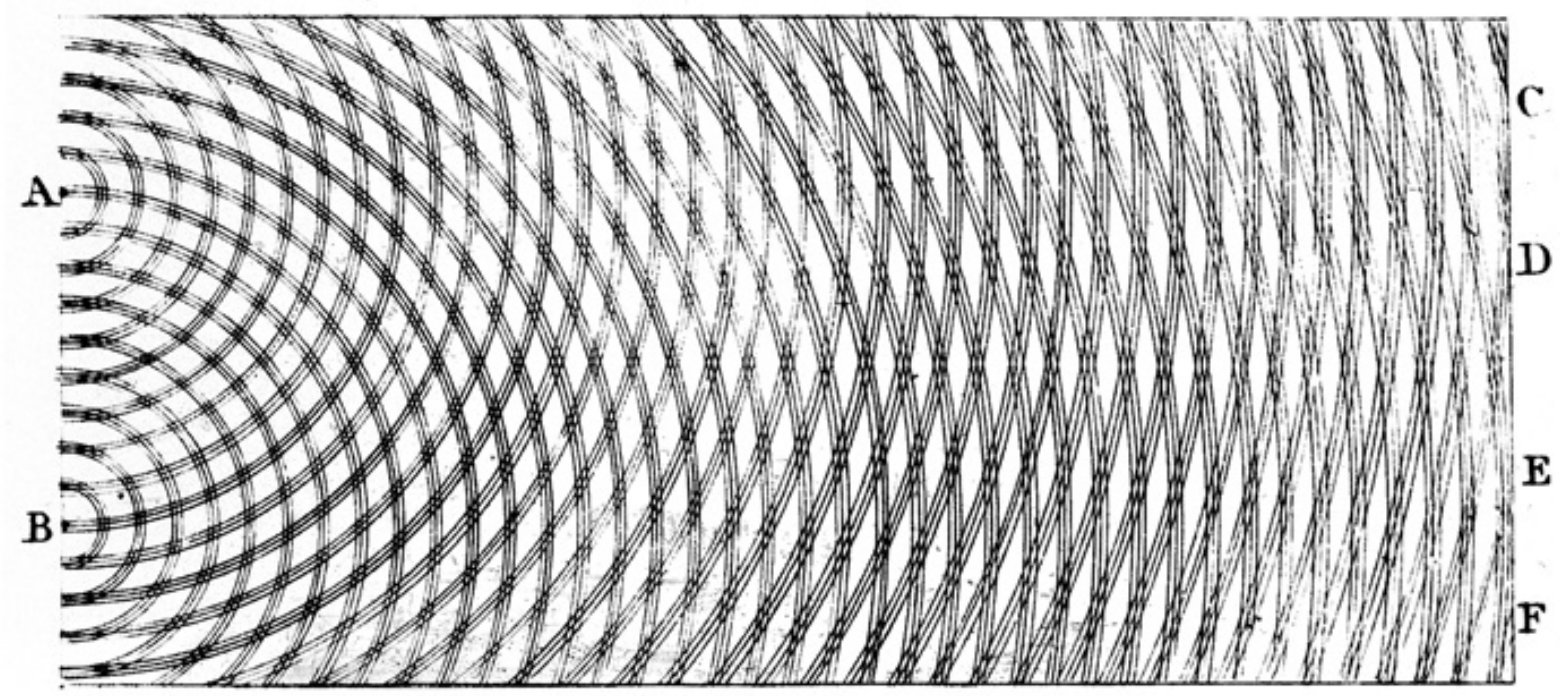


Figura 3. Aparatos como los utilizados por Fizeau en su experimento

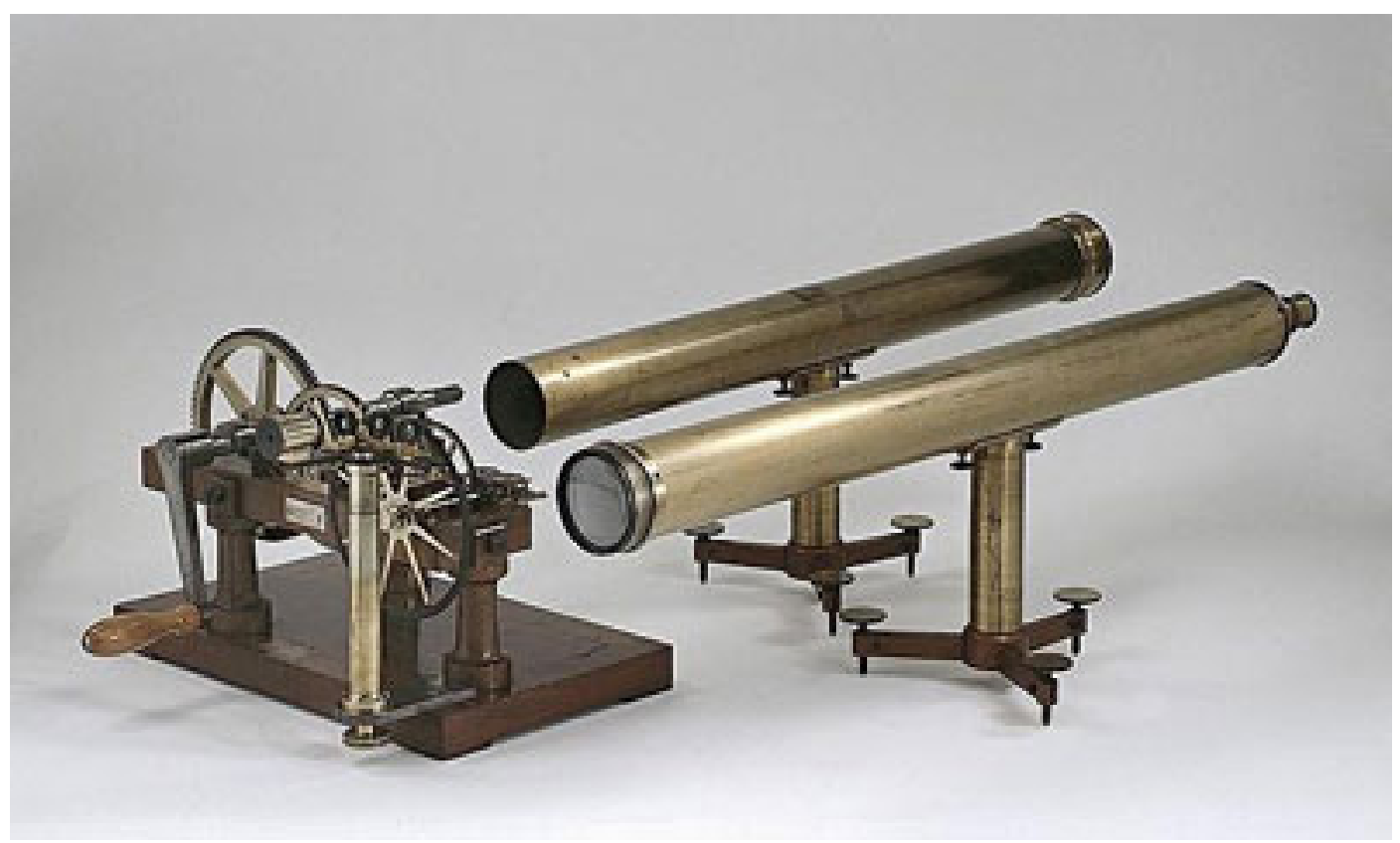

\section{ELECTRICIDAD Y MAGNETISMO}

El fenómeno consistente en que un objeto atrae a otros más pequeños y ligeros al ser frotado, se conocía al menos desde la antigua Grecia. Tales de Mileto dejó constancia de haber observado esta propiedad

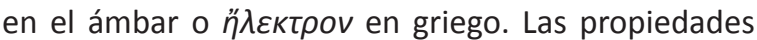
atractivas y repulsivas de la magnetita, llamada así por la región de Magnesía, eran conocidas ya desde antes. A principios de siglo XVII las observaciones sobre electricidad y magnetismo se habían ido recopilando en sucesivos escritos. El médico y físico inglés William Gilbert diferenció entre sustancias eléctricas e ineléctricas. Las primeras tendrían la capacidad de atraer objetos después de ser frotadas y las segundas no. En cuanto al magnetismo describió la existencia de los dos polos de un imán y que los polos iguales se repelen mientras que los distintos se atraen. También escribió sobre la imposibilidad de aislar un polo magnético partiendo sucesivas veces un imán. Gilbert mostraba más interés por los hechos y los experimentos que por la pura especulación. Mencionaba por ejemplo los patrones dibujados por las limaduras de hierro sobre un papel en las proximidades de un imán. Después de estudiar los imanes esféricos concluía que la tierra se comportaba como uno de ellos. Aunque muchos creían que electricidad y magnetismo estaban relacionados se estudiaron como fenómenos separados durante siglos.

\section{Electricidad}

En el siglo XVII se produjo un invento que posibilitó una sucesión de experimentos que permitieron el avance en el conocimiento de los fenómenos eléctricos. El alemán Otto von Guericke construyó la primera máquina electrostática por frotación. Consistía en una esfera de azufre atravesada por una barra de hierro. Cuando se colocaba la mano sobre ella y se hacía girar unas cuantas veces podía atraer todo tipo de objetos. En el siglo siguiente el inglés Stephen Gray realizó gran número de experimentos con este aparato y llegó a la conclusión que aquellas sustancias como los metales, que al ser frotadas no se electrizaban, eran conductoras del efluvio eléctrico. Logró transmitir electricidad a través de un alambre una distancia de 100 metros.

En 1733, el francés Charles François de Cisternay du Fay publicó un trabajo donde identificaba dos tipos de cargas eléctricas distintas. Según su naturaleza los cuerpos se podían cargar de electricidad vítrea o resinosa. Los que habían adquirido electricidades de tipos distintos se atraían y los que habían adquirido el mismo tipo de electricidad se repelían. En 1785, Charles de Coulomb dio a conocer una ley matemática que cuantificaba la fuerza eléctrica con que se atraían o repelían dos cuerpos cargados. Dependía de la carga eléctrica de los cuerpos y del inverso del cuadrado de su distancia, a modo de la ley de la gravitación de Newton. Posteriormente fue establecida una ley análoga para la fuerza magnética. 
Por otro lado en la década de 1740 el holandés Pieter van Musschenbroek dio a conocer la botella de Leyden, el primer condensador eléctrico. Consistía en una botella con un tapón atravesado por una varilla metálica sumergida en agua. Si se ponía en contacto el extremo exterior de la varilla con una máquina electroestática, el agua del interior se cargaba. Si se acercaba el extremo exterior de la varilla a un cuerpo no aislado se producía una chispa y una descarga eléctrica. Pocos años después el polifacético norteamericano Benjamín Franklin (Summers, 2002) demostró que los rayos eran descargas electroestáticas como las de la botella en su famoso experimento de la cometa. También formuló la teoría del fluido único y clasificó las sustancias en positivas o negativas según si tenían exceso o defecto de fluido eléctrico.

\section{Electromagnetismo}

Antes del siglo XIX muchos pensadores habían sospechado que electricidad y magnetismo eran dos fenómenos relacionados entre sí. Una vez más, hubo que esperar a una innovación técnica para afianzar los cimientos de esa sospecha. Sucedió en 1800 gracias a la pila inventada por el científico italiano Alessandro Volta. Pocos años atrás, el médico Luigi Galvani había observado que, al tocar una pata de rana con dos metales distintos en contacto entre sí, esta se movía. Identificó este fenómeno como electricidad animal. Volta, después de repetir los experimentos, concluyó que el fenómeno tenía su origen en los metales y no en la rana. Ideó un dispositivo para realizar descargas eléctricas que consistía en discos de zinc y plata apilados en batería entre los que se colocaba cartón empapado en agua salada. Resultó que producía una tensión eléctrica permanente, lo cual permitió tener circuitos con corriente eléctrica continua. Este hecho fue un punto de inflexión en el conocimiento de la electricidad ya que posibilitó muchos y nuevos experimentos que permitieron relacionarla con el magnetismo.

En 1819, el danés Hans Christian Ørsted (Pérez y Varela, 2003) observó que al colocar una brújula cerca de un cable por el que circulaba corriente eléctrica su aguja sufría una desviación tendiendo a ponerse perpendicular a él. Meses más tarde, el francés AndréMarie Ampère (Pérez y Varela, 2003) estudió la relación que regia este fenómeno. La fuerza magnética alrededor de un cable era proporcional a la intensidad de corriente eléctrica que circulaba por él. También comprobó que existía interacción entre dos conductores paralelos. Si la corriente iba en la misma dirección se atraían, en dirección contraria se repelían. Observó que un cable enrollado en espiral (bobina) por el que circulaba corriente se comportaba igual que un imán. Esto le llevó a suponer que el magnetismo era una manifestación del movimiento de las cargas eléctricas y que no podía existir un polo magnético aislado. Poco después el inglés William Sturgeon construyó un electroimán regulable. Consistía en un trozo de hierro envuelto en un cable por el que podía circular corriente eléctrica. Con la fuerza magnética que producía podía levantar $4 \mathrm{~kg}$. Su desarrollo posibilitó el telégrafo y el motor eléctrico ya que podía convertir la corriente eléctrica en fuerza motriz.

Por aquel entonces, Carl Friedrich Gauss, después de múltiples mediciones topográficas y estudios de las superficies curvas, estaba desarrollando las ideas de Laplace sobre las acciones gravitatorias terrestres. Para el caso análogo de las acciones generadas por cargas eléctricas se tenía que la suma del flujo que atravesaba una superficie cerrada era proporcional a la cantidad de electricidad o carga que albergaba en su interior. Aplicando las leyes de la dinámica de fluidos al caso de una carga puntual la intensidad de flujo en un punto variaba con la ley del inverso del cuadrado de la distancia. Esta expresión era pues equivalente a la ley de Coulomb para las fuerzas electroestáticas. Un tratamiento similar para las acciones magnéticas daba que la suma del flujo que atravesaba una superficie cerrada era cero. Esto se explicaba porque el fluido magnético se distribuía en curvas cerradas que entraban y salían por igual de cualquier superficie cerrada. Era equivalente a afirmar la imposibilidad de un polo magnético aislado.

En este punto de la historia es cuando aparece el científico inglés Michael Faraday (Díaz-Hellín, 2001). Después de repetir los experimentos de $\varnothing$ rsted, Ampère y hacer experimentos propios, comprobó la producción de fuerza magnética capaz de generar movimiento continuo alrededor de un cable por el que circula corriente eléctrica. Se propuso entonces estudiar si se podía también generar corriente eléctrica a través del magnetismo. En 1831, el año en que nació Maxwell, después de realizar múltiples experimentos llegó a la conclusión que sí. Se podía inducir corriente eléctrica en un conductor si se sometía a los efectos de un dispositivo magnético en movimiento.

Uno de los experimentos que realizó Faraday consistió en la construcción de un aparato formado por un anillo de hierro dulce con cable enrollado en cada uno de sus dos lados. Uno de estos cables podía abrir y cerrar un circuito con una pila. El otro cerraba un circuito con un dispositivo detector de corriente eléctrica. Observó que solo se detectaba electrici- 
Figura 4. Esquema de la ley de Gauss para flujo eléctrico

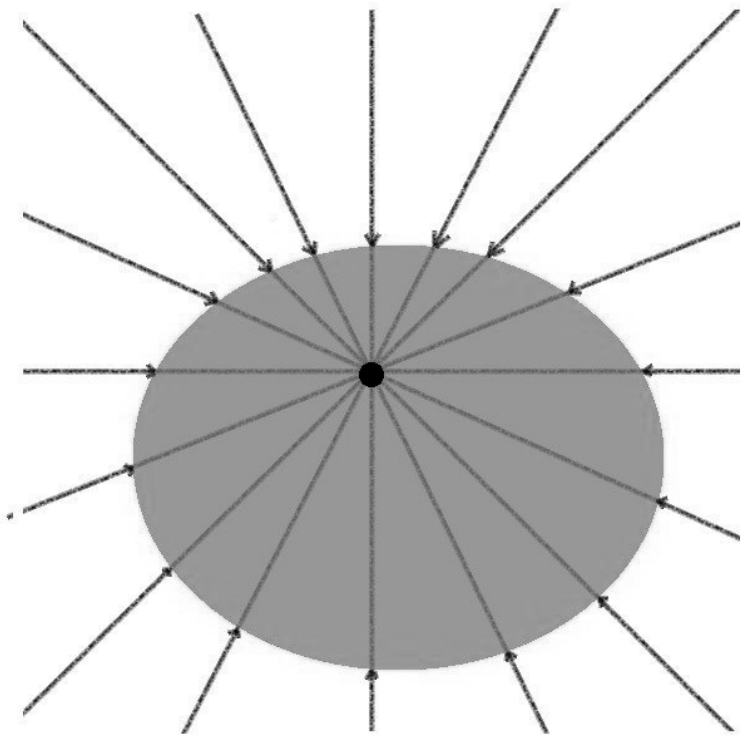

Figura 5. Esquema de la ley de Gauss para flujo magnético

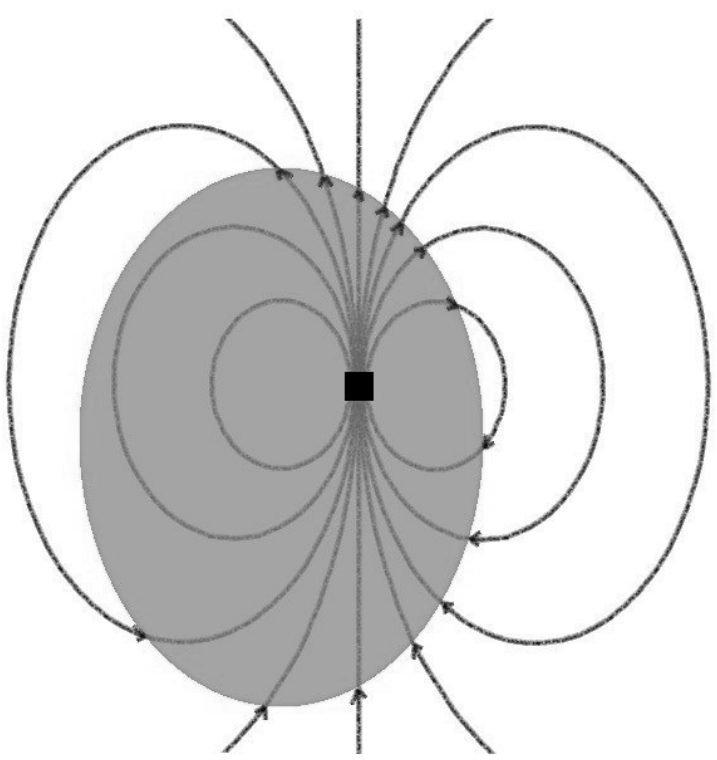

dad en el segundo circuito en el momento en que se conectaba o desconectaba la pila. Creyendo que la corriente que aparecía era inducida por la variación de los efectos magnéticos producidos por el primero, realizó el experimento siguiente: introdujo un imán en el interior de un cable enrollado o bobina y observó que solamente se generaba corriente en esta cuando el imán era movido. Quedaba demostrada así la posibilidad de generar corriente eléctrica en un circuito a través del magnetismo.
Figura 6. Esquema de la ley de Ampère

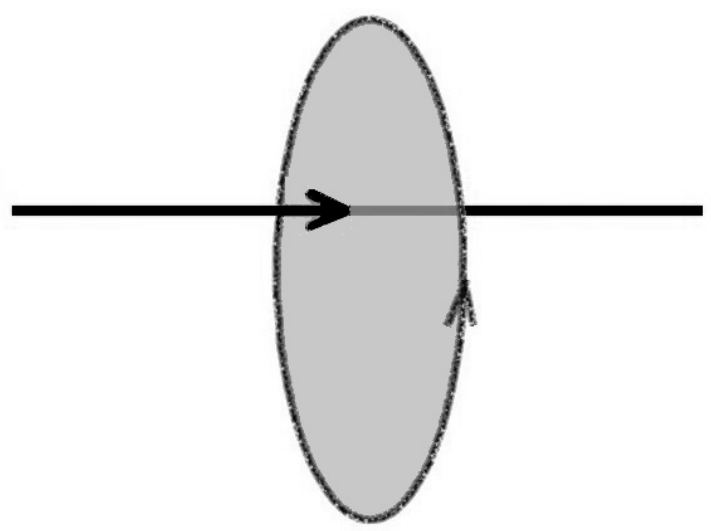

Figura 7. Experimento de Faraday sobre la inducción

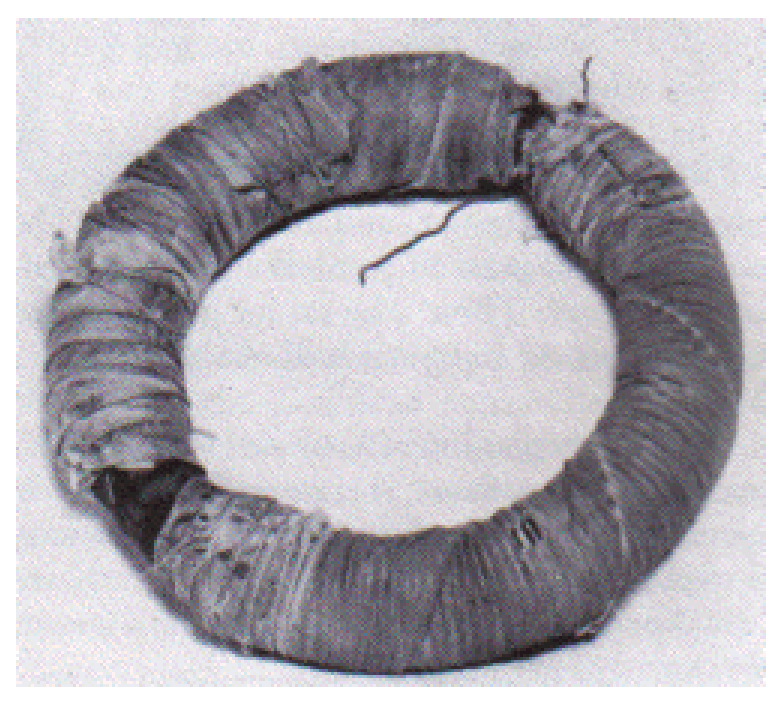

No contento con eso Faraday aún dio un paso más construyendo una dinamo. Se trataba de un dispositivo capaz de generar corriente eléctrica continua. Consistía en un disco de cobre giratorio colocado entre los extremos de un imán en herradura. El disco estaba unido a un circuito mediante dos contactos deslizantes. En el circuito había un dispositivo medidor de corriente eléctrica que indicaba la presencia de corriente continua mientras el disco giraba. Hasta ese momento se podía generar fuerza motriz a través de 
corriente eléctrica, pero a partir de entonces se pudo generar también corriente eléctrica a través de fuerza motriz.

El descubrimiento de la inducción electromagnética dejaba la puerta abierta al dominio de la energía eléctrica tal como lo conocemos actualmente. Poco después de conocer el experimento de la dinamo, el joven fabricante de instrumentos parisino Hippolyte Pixii, construyó un generador eléctrico donde lo que giraba era el imán. Este primer generador de corriente alterna contenía ya el mecanismo básico para el posterior desarrollo de todas las centrales de generación de energía eléctrica a gran escala.

\section{LAS LÍNEAS DE FUERZA}

La idea de las fuerzas a distancia propagadas por un fluido sutil existía ya desde la antigua Grecia y siguió apareciendo de forma recurrente, por ejemplo en el siglo XVII con los vórtices de Descartes. Cuando Newton postuló su ley de la gravitación universal dando sencillas fórmulas para describir la acción de fuerzas a distancia, se popularizó la percepción de que estas actuaban de forma instantánea. Parece que el propio Newton creía que las fuerzas se propagaban por algún medio indetectable, pero la potencia y sencillez de sus ecuaciones permitía omitir esta cuestión. En la visión mecanicista que se desarrolló a partir de sus ideas no se necesitaba para nada la existencia de un medio de propagación. En el caso de las fuerzas eléctricas la ley del inverso del cuadrado dada a conocer por Coulomb a finales del siglo XIX actuó de forma similar. Sin embargo el mecanismo de propagación a distancia seguía oculto.

En el siglo XIX la atención estaba centrada principalmente en las fuerzas que actuaban a distancia entre dos cuerpos, aparentemente de forma instantánea, omitiendo el espacio intermedio. En el caso de las fuerzas gravitatorias y eléctricas las ecuaciones funcionaban perfectamente. En el caso de las fuerzas magnéticas con un comportamiento más complejo, se hacía necesario un tratamiento distinto. Es entonces cuando apareció Faraday con su modelo de líneas de fuerza. Hijo de un herrero probablemente había visto en su infancia los patrones de las limaduras de hierro provocados por un imán. Un fenómeno que estaba documentado desde hacía siglos. Las líneas de fuerza eran un artilugio que utilizó para describir el estado que generaba un imán en su entorno y fueron el germen de la idea de campo.

Desde finales del siglo XVIII ya se habían ido desarrollando formalismos matemáticos para el flujo gra- vitatorio, eléctrico y magnético gracias a personajes como Laplace, Ampère, Biot, Savart, Poisson, Gauss o Weber. La idea de campo estaba ya en el aire cuando Faraday propuso su modelo de las líneas de fuerza. Lo que realmente cambió fue la percepción de que no se trataban solamente de un formalismo matemático útil, sino un estado que producían los objetos en el espacio de su alrededor. Las líneas de fuerza eran las curvas cuya tangente en un punto proporcionaba la dirección de la fuerza que sufriría un objeto que interaccionase con el que las causaba. En el caso de un objeto cargado eléctricamente, se trataba de rectas con un extremo en el centro del objeto cuyas fuerzas disminuían en intensidad con la ley del inverso del cuadrado de la distancia y variaban su sentido según el tipo de carga. En un imán iban desde un polo hacia el otro tal como mostraban los patrones de las limaduras de hierro y se unían por el otro extremo formando circuitos cerrados.

Las aproximaciones de Faraday eran totalmente intuitivas y basadas en los experimentos. Precisamente esto es lo que las hacía más interesantes, ya que se enfrentaban al problema desde un punto de vista más fresco y alejado de las ataduras del conocimiento académico. Sin embargo su falta de formación en matemáticas y la dificultad del modelo geométrico que había ideado no le permitieron encontrar unas fórmulas adecuadas para describirlo. Aquí es donde entra en la historia Maxwell. Después del esfuerzo de rigor que le había supuesto adaptar su potente intuición geométrica a la disciplina matemática necesaria para afrontar sus exámenes de graduación en la Universidad de Cambridge, estaba en condiciones de abordar el problema. Empezó pues a buscar analogías que le permitieran encontrar unas formulas sencillas que describieran el comportamiento de los campos eléctrico y magnético.

Con el desarrollo del cálculo diferencial y los nuevos descubrimientos científicos, las analogías entre distintos fenómenos con un tratamiento matemático idéntico estaban adquiriendo cada vez más complejidad. Uno de los que habían iniciado este camino era William Thompson con el que entonces Maxwell tenía una cordial relación y mantenía correspondencia. Desde joven, Thompson venía estudiando la analogía entre la variación en el espacio de las fuerzas electroestáticas y el gradiente de calor en un sólido. Siguiendo su ejemplo, Maxwell intentó concebir un modelo propio que se comportase de manera análoga a las líneas de fuerza y permitiese a su vez ser descrito con ecuaciones matemáticas sencillas. 
Figura 8. Patrones de limaduras de hierro bajo el efecto de un imán

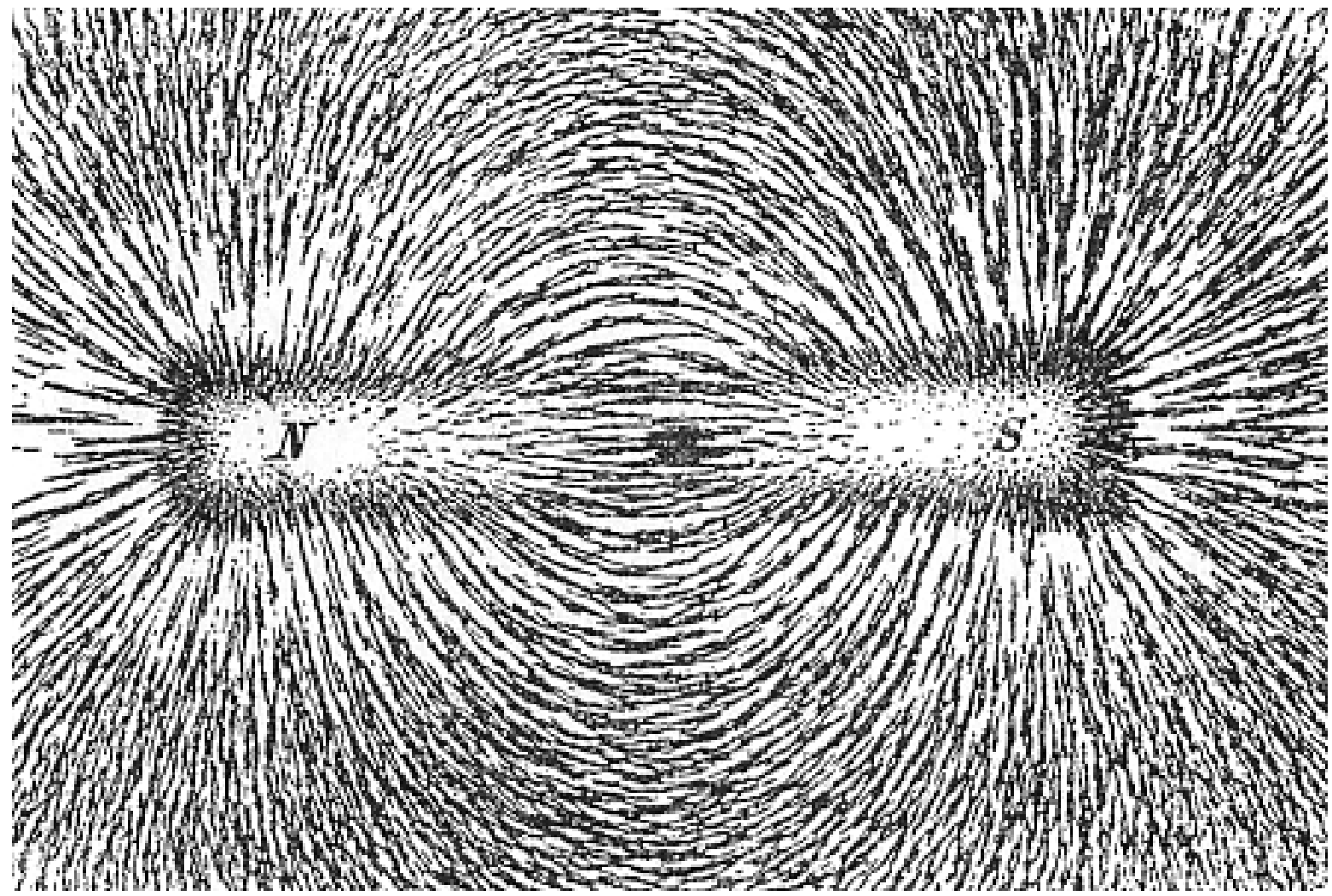

La analogía que ideó Maxwell consistía en imaginar un fluido incompresible y sin peso que ocupaba el espacio. La velocidad y dirección del fluido en un punto se correspondían con la intensidad y dirección de la fuerza. La velocidad y dirección del fluido eran distintas en distintos puntos del espacio pero no variaban con el tiempo. Las curvas que representaban los caminos de las partículas y que por tanto indicaban la dirección del movimiento eran llamadas líneas de movimiento del fluido. Para elaborar un modelo espacial consideraba una superficie que las cortara todas. Imaginaba sobre ella otros dos sistemas de líneas, de tal manera que quedara dividida en cuadriláteros por los que pasaba una unidad de fluido por unidad de tiempo. Entonces imaginaba líneas de movimiento de fluido desde cada uno de los puntos de los bordes de estos cuadriláteros, de tal forma que el espacio quedaba dividido por superficies tubulares entre las que no había intercambio de fluido. Como este era incompresible, cualquier sección de un tubo que se tomara sería atravesada también por una unidad de fluido por unidad de tiempo. Obtuvo así una construcción geométrica que dividía completamente el espacio en tubos unitarios. La velocidad del fluido en un punto venia definida por la dirección del tubo y el inverso de la superficie de su sección.
Las cualidades eléctricas y magnéticas de los distintos materiales se correspondían con una distinta resistencia al movimiento del fluido en distintos medios. Esto permitía la representación en el modelo de los materiales conductores y aislantes. La resistencia era proporcional a la velocidad del fluido en cada punto. Para que esta última no disminuyese con el tiempo, era necesaria una diferencia de presión que contrarrestase los efectos de la resistencia. El gradiente de presión, representaba a la intensidad de campo. En el caso eléctrico, era producido por fuentes o sumideros que representaban a los dos tipos de cargas. Las fuerzas de repulsión o atracción eran debidas al exceso o defecto de fluido. Aplicando esta analogía obtuvo unas ecuaciones que, desarrolladas, no solo daban la ley del inverso del cuadrado de las fuerzas a distancia, sino que también daban cuenta de otros fenómenos eléctricos como la conducción a través de circuitos. Plasmó sus conclusiones en la primera parte de su artículo "On Faraday's Lines of Force" (presentado en diciembre de 1855). Pero aún le faltaba encontrar ecuaciones para los fenómenos más complicados como la inducción electromagnética. 
Para explicar la inducción electromagnética, Faraday se había valido del concepto de estado electrotónico generado por un imán o un circuito eléctrico. Al alterar este estado por movimiento relativo respecto a otro circuito adyacente, se producía en este último una circulación de corriente eléctrica. Maxwell abordó matemáticamente la descripción de este fenómeno en la segunda parte de su artículo que tituló "On Faraday's "Electro-tonic State"”" (presentado en febrero de 1856). Para hacerlo no le bastó con un modelo tridimensional y ecuaciones sencillas, sino que necesitó unas herramientas más potentes. Así que utilizó las novedosas técnicas de cálculo vectorial avanzado que acababa de aprender en la preparación de sus exámenes de graduación en Cambridge.

El concepto de vector estaba ya implícito en la mecánica definida por Newton a finales del siglo XVII. Las operaciones vectoriales (suma y productos escalar y vectorial) se vinieron desarrollaron en los siglos posteriores y fueron finalmente expuestas de forma sencilla en 1844 por el alemán Grassmann. Los últimos avances en la materia se habían desarrollado aplicando geometría diferencial gracias a dos profesores y amigos de Maxwell: George Stokes y William Thompson. Todo el desarrollo posterior del cálculo vectorial tuvo una relación íntima con el de de la idea de campo electromagnético.

Teniendo en cuenta las leyes conocidas que relacionaban electricidad y magnetismo, Maxwell estudió qué pasaría en una pequeña porción del espacio. Utilizando el cálculo vectorial obtuvo una magnitud vectorial que describía perfectamente el estado electrotónico de Faraday. Si tenía un valor constante no tenía efecto alguno, pero su variación implicaba la aparición de fuerzas eléctricas y magnéticas. La notación que utilizó dista un poco de la actual ya que para cada magnitud vectorial escribía las tres ecuaciones de las componentes en los ejes espaciales.

Las dos partes del artículo sobre las líneas de fuerza fueron leídas en la Cambridge Philosopical Society en diciembre de 1855 y febrero 1856 y publicadas en sus Transactions años más tarde (Maxwell, 1864). Para Maxwell con veinticuatro años este trabajo sería solo una buena base para construir una teoría más completa y elaborada. Sus aportaciones más importantes para la comprensión y descripción de los fenómenos electromagnéticos estaban aún por llegar. Sin embargo, el artículo por sí solo tuvo gran trascendencia. Para darse cuenta solo hace falta ver lo que le escribió el propio Faraday después de haberlo leído:
"Recibí tu artículo, y te lo agradezco mucho. No me aventuro a agradecerte lo que has dicho sobre las "Líneas de Fuerza", porque sé que lo has hecho por el interés de la verdad filosófica; pero debes suponer que es un trabajo muy gratificante para mí, y me da mucho coraje para reflexionar. Estaba al principio casi aterrorizado cuando vi tanta fuerza matemática hecha enlazar con el tema y después maravillado por ver que el tema lo aguantaba tan bien."

\section{LA LUZ ES UNA ONDA ELECTROMAGNÉTICA}

En el otoño de 1860 Maxwell se disponía a iniciar su nueva etapa como profesor en el King's College de Londres. Después de los cuatro años en Aberdeen preparando el curso de filosofía natural, había desarrollado una amplia visión global del estado de la física. Había analizado e incluso ayudado en el desarrollo de las teorías e hipótesis más novedosas de su tiempo situándose en primera línea del conocimiento científico. Cabe destacar que los trabajos realizados sobre Saturno y los gases le permitieron profundizar sus conocimientos de mecánica y elasticidad. Lo cual le resultaría de muchísima utilidad en sus posteriores aproximaciones al fenómeno electromagnético. Con veintinueve años estaba a un paso de realizar su aportación más importante a la historia de la ciencia.

\section{El modelo de los vórtices rotatorios}

Si bien desde su anterior artículo sobre las líneas de fuerza habían pasado ya cinco años, Maxwell siempre tuvo presente el fenómeno electromagnético. Continuó con la analogía del fluido, pero esta vez poniendo la atención en las partes de que estaba formado. Consideró un medio constituido por vórtices cuya rotación provocaba un estado tensional equivalente a las líneas de fuerza magnética. Para que no hubiera rozamiento entre ellos introdujo unas capas intermedias de pequeñas partículas, que si se consideraban de naturaleza eléctrica podían dar cuenta de las corrientes. Todas estas reflexiones cristalizaron en un artículo titulado "On Physical Lines of Force" (Maxwell, 1861a, 1861b, 1862a, 1862b). Este fue inicialmente publicado en dos partes en marzo y abril de 1861 en The London, Edinburg and Dublín Philosophical Magazine and Journal of Science. En ellas trataba de los fenómenos referentes a las fuerzas magnéticas, las corrientes eléctricas y la relación entre ambos fenómenos.

En la Parte 1 (Maxwell, 1861a), titulada "The Theory of Molecular Vortices applied to Magnetic Phenome$n a^{\prime \prime}$, proseguía con su analogía del fluido analizando las partes de que estaba formado desde un punto de vista 
mecánico. Intentaba elaborar un modelo que permitiera extraer consecuencias análogas a todos los fenómenos eléctricos y magnéticos conocidos hasta entonces: "Mi objetivo en este artículo es aclarar el camino para la especulación en esta dirección, investigando los resultados mecánicos de ciertos estados de tensión y movimiento en un medio, y comparándolos con los fenómenos observados del magnetismo y la electricidad."

Maxwell estaba perfectamente familiarizado con los estados de tensión internos de los cuerpos, es decir las acciones y reacciones entre sus partes consecutivas. Desde el establecimiento de las tres leyes básicas de la mecánica por Newton, se habían ido encontrando relaciones matemáticas cada vez más complejas para los estados de tensión internos de los cuerpos (momento flector, esfuerzo de cizalla y elasticidad transversal). Uno de los conceptos centrales junto a las fuerzas de acción y reacción era la elasticidad, la propiedad de aquellos cuerpos que tendían a conservar su forma. Esta propiedad fue inicialmente descrita por Robert Hooke para los muelles. Según la ley que lleva su nombre, el alargamiento de un muelle es proporcional a la fuerza que ejerce para volver a su longitud inicial.

Maxwell empezó su analogía considerando las líneas de fuerza magnética como el estado de tensión en un medio. Este venía definido por tres direcciones ortogonales de presión en cada punto. Dos de ellas eran iguales y la tercera un eje de simetría, en este caso con la presión mínima ya que la tensión a lo largo de las líneas magnéticas se correspondía con una tracción. Este estado podía explicarse por la existencia de vórtices rotatorios que tenderían a contraerse en el eje de giro y expandirse en el plano perpendicular por la fuerza centrífuga. La rotación se correspondía con el carácter bipolar de las fuerzas magnéticas ya que había dos sentidos posibles. Como convención escogió el de las agujas del reloj mirando en dirección norte.

Después de estudiar el comportamiento mecánico de uno de estos vórtices, buscó la relación de su velocidad de rotación y densidad con las tensiones provocadas en el medio. La velocidad en su ecuador debía ser proporcional a la intensidad de la fuerza magnética y su densidad a la capacidad de inducción magnética del medio. Por ejemplo hierro y níquel con una alta capacidad de inducción se considerarían medios con una densidad elevada.

En la Parte 2 (Maxwell, 1861b), titulada "The Theory of Molecular Vortices applied to Electric Currents", empezaba preguntándose qué era lo que ponía a los vórti- ces en movimiento y terminaba preguntándose por la naturaleza de las corrientes eléctricos: "Sabemos que las líneas de fuerza están afectadas por corrientes eléctricas, y sabemos la distribución de esas líneas sobre una corriente; de tal forma que de la fuerza podemos determinar la cantidad de corriente. Asumiendo que nuestra explicación de las líneas de fuerza a través de vórtices moleculares es correcta, ¿por qué indica una distribución particular de vórtices una corriente eléctrica? Una respuesta satisfactoria a esta pregunta nos acercaría un buen camino hacia la de otra muy importante, "¿Que es una corriente eléctrica?"”

El hecho de que dos vórtices adyacentes rotaran en la misma dirección suponía un problema ya que sus velocidades en los puntos de contacto tenían direcciones contrarias y su rozamiento ocasionaría una desaceleración. Para solucionarlo introdujo unas capas de pequeñas partículas intermedias a su alrededor que rotaban en dirección contraria a modo de idle wheels o ruedas pasivas. Las ruedas pasivas normalmente tenían un eje fijo, pero en algunos mecanismos como los trenes epicíclicos o los limitadores de velocidad su eje tenía cierto margen de movimiento. Este movimiento dependía de las distintas velocidades de las ruedas principales. Siguiendo con el modelo, se podía imaginar que las partículas dispuestas en las capas intermedias podían tener movimiento de traslación. Si se las consideraba como partículas eléctricas su movimiento se correspondería con una corriente.

La representación de la corriente eléctrica como movimiento de partículas, también ofrecía una explicación a su interacción con el campo magnético. Si se imaginaba un circuito recorrido por partículas eléctricas, su movimiento produciría la rotación de los vórtices circundantes. Esta rotación se correspondería con una línea de fuerza magnética alrededor del circuito tal como las descritas según la ley de Ampere.

El modelo también era capaz de explicar el fenómeno de la inducción electromagnética. Si se imaginaba una configuración equivalente a un campo magnético uniforme, las partículas de las capas intermedias solamente rotaban. Pero si se imaginaba una configuración equivalente a un campo magnético variable, las partículas también se podían desplazar ya que en uno de sus lados los vórtices giraban con mayor velocidad. Este movimiento de partículas era equivalente a una corriente eléctrica. Sin embargo solo se manifestaba como tal en aquellos medios cuyas partículas gozaban de un cierto grado de libertad, es decir en los medios conductores con baja resistencia eléctrica. En los medios aislantes con alta 
resistencia, la presión de unas partículas sobre otras se manifestaría en forma de fuerza electroestática. Sin embargo el mecanismo por el cual sucedía este último fenómeno aun no quedaba explicado.

Para estudiar con detalle el fenómeno de la inducción de una corriente eléctrica escogió el campo magnético variable generado por la aparición y desaparición de otra corriente. En la Figura 9 (aparecida en el artículo como Figura 2), la corriente inicial venía representada por la traslación de partículas desde $A$ hasta B. Si se iniciaba de repente, los vórtices inmediatamente contiguos g-h empezaban a girar mientras los siguientes k-I permanecían en reposo. La línea de partículas intermedia $\mathrm{p}-\mathrm{q}$ se desplazaría hacia la izquierda representando una corriente inducida en dirección contraria a la inicial. Este movimiento de partículas haría girar los siguientes vórtices k-l hasta igualar su velocidad con los primeros g-h. En ese momento las partículas intermedias $p-q$ cesarían su desplazamiento y simplemente rotarían, con lo cual la corriente inducida se terminaría. De forma similar se explicaba la corriente inducida en el mismo sentido que la inicial cuando esta desaparecía. Este proceso se repetía en las sucesivas líneas de vórtices y partículas en todas las direcciones del espacio. Quedaba así aclarado dentro del modelo por qué era la variación del campo magnético y no el propio campo lo que inducía las corrientes eléctricas. Ya solo le faltaba incorporar las fuerzas electroestáticas.

Figura 9. Esquema aparecido en "On Physical Lines of Force" como Figura 2

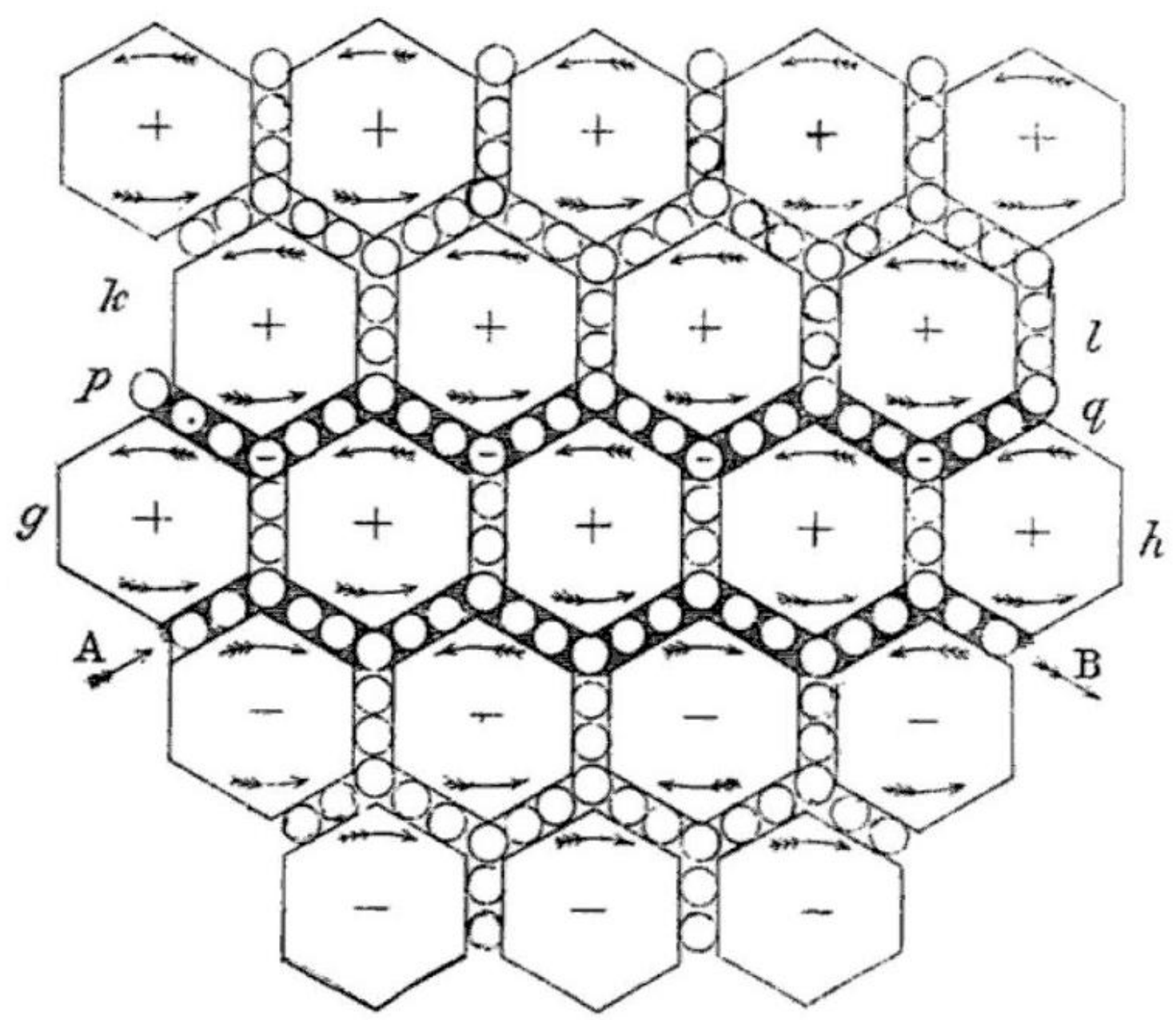




\section{Las ondas electromagnéticas}

Tras la publicación de las partes 1 y 2 de su artículo "On Physical Lines of Force", Maxwell siguió trabajando sobre el modelo de vórtices rotatorios, para incorporar las fuerzas electroestáticas. Ese mismo verano de 1861 en Glenlair, tuvo una idea que se revelaría muy fructífera y tendría importantes consecuencias. Se trataba de considerar un medio elástico e identificar las fuerzas provocadas por la elasticidad transversal de los vórtices con las fuerzas electroestáticas. El desarrollo de esta nueva idea le llevó a la convicción que los fenómenos electromagnéticos se propagaban en forma de ondas transversales. Al ser similares a las ondas lumínicas, intuyó que quizás ambos fenómenos tenían lugar en el mismo medio. Cuando llegó a Londres en otoño, después de consultar un par de libros y realizar varios cálculos comprobó que su intuición tenía fundamento. La velocidad de propagación de los fenómenos electromagnéticos en forma de ondas transversales que le permitía calcular su modelo coincidía con la velocidad de la luz medida con medios ópticos. Todas estas ideas fueron publicadas como las partes 3 y 4 del mismo artículo en diciembre de 1861 y febrero de 1862.

En la Parte 3 (Maxwell, 1862a), titulada "The Theory of Molecular Vortices applied to Statical Electricity", trataba las fuerzas electroestáticas. En los materiales aislantes, las partículas eléctricas estaban ligadas a los vórtices (o células como también les llama en esta parte) rotantes de tal manera que no podían pasar de uno a otro. Si se dotaba a las células de elasticidad, se deformarían ante la presión de las partículas eléctricas al desplazarse. Esta distorsión y su tendencia a volver a la forma original, permitirían la explicación dentro del modelo de los fenómenos de atracción y repulsión de los cuerpos cargados eléctricamente.

El artículo empezaba estudiando las condiciones de equilibrio de una de las células, tratándola como una esfera elástica cuya superficie estaba expuesta a fuerzas tangenciales y normales. Considerando que la elasticidad del medio dependía de fuerzas actuando entre pares de células, obtenía una expresión para la intensidad de campo eléctrico ${ }^{2}$. Dependía de la elasticidad transversal del medio y del desplazamiento de las partículas eléctricas. Este pequeño movimiento de las partículas constituía también una corriente de desplazamiento. La añadió a la fórmula de Ampère que relacionaba la corriente eléctrica ordinaria con la intensidad magnética. Resultaron tres ecuaciones en sendas direcciones del espacio que eran la forma primitiva de lo se llamaría ley de Ampère-Maxwell.
Por otro lado la variación temporal de la carga ${ }^{3}$ en un punto se podía expresar en función de la variación espacial de la corriente en ese punto. Utilizando las tres ecuaciones que acababa de encontrar se podía definir la carga únicamente en función de la elasticidad transversal del medio y de la variación espacial de la intensidad de campo eléctrico.

Seguidamente calculaba la fuerza electroestática entre dos cuerpos cargados buscando la energía debida al desplazamiento eléctrico que provocaban. Utilizando la expresión para la carga que acababa de encontrar obtenía una ecuación que relacionaba esta energía con la carga de uno de los cuerpos y el potencial del otro. Igualando su variación al trabajo necesario para separar ambos cuerpos obtenía una expresión para la fuerza electroestática. Esta dependía de las cargas de los cuerpos, el inverso del cuadrado de su distancia y la elasticidad transversal del medio. Conjugando esta expresión de la fuerza electroestática con la de la fuerza magnética generada por las mismas cargas en movimiento, obtenía un interesante resultado. La elasticidad transversal del medio se podía expresar en función del ratio entre las unidades electroestática y electrodinámica de medición de carga.

En la segunda parte del artículo había explicado la inducción electromagnética a través de la sucesiva puesta en movimiento de los vórtices (o células) contiguos propagada con una velocidad finita. Si las células gozaban de elasticidad, era previsible que este proceso avanzara en forma de ondas. Al transmitirse simultáneamente el movimiento de las partículas eléctricas y la rotación de las células que describían las líneas de fuerza magnética, se trataría de ondas electromagnéticas. Por otro lado tanto el desplazamiento de partículas como la distorsión de células se producían en una dirección perpendicular a la de propagación. De la teoría de ondas transversales se desprendía que la velocidad de propagación en un medio era la raíz cuadrada del ratio entre su elasticidad transversal y su densidad.

En la primera parte del artículo había definido la analogía entre la densidad de los vórtices y su capacidad de inducción magnética cuyo coeficiente en el vacío era la unidad. Conjugando la expresión de la velocidad de propagación de las ondas transversales con la que acababa de encontrar para la elasticidad transversal del medio obtenía un resultado muy interesante. La velocidad de propagación de las ondas transversales en el medio electromagnético era igual al ratio entre las unidades electrodinámica y electroestática de medición de carga. Este ra- 
tio había sido determinado experimentalmente por Rudolf Kohlrausch y Wilhelm Weber (Kohlrausch y Weber, 1857).

Weber había trabajado años atrás en Gottingen junto a Gauss en el establecimiento de un sistema de unidades electromagnéticas (Assis, Reich y Wiederkehr, 2002). Había definido la unidad de intensidad de corriente eléctrica en función de la unidad de fuerza magnética por unidad de longitud y las leyes electromagnéticas. Para hacerlo había asumido la antigua hipótesis según la cual los dos tipos de electricidad vitrea (positiva) y resinosa (negativa) constituían dos fluidos circulando en ambas direcciones al mismo tiempo ${ }^{4}$. A partir de la unidad de intensidad por unidad de tiempo definía la unidad electrodinámica de medición de carga. Por otro lado se podía definir también la unidad electroestática de medición de carga en función de la unidad de fuerza eléctrica y la unidad de longitud según la ley del inverso del cuadrado de la distancia. El análisis dimensional mostraba que el ratio entre ambas mediciones era una velocidad. Presumiblemente la de propagación de los fenómenos electromagnéticos.

El experimento de Kohlrausch y Weber se dividía en dos partes (Ebel, 1993; Assis, 2003). En la primera se cargaba una botella de Leyden con un generador electroestático. Se pasaba una porción de esta carga a una esfera metálica con capacidad conocida y convenientemente aislada. Después se pasaba la mitad de la carga de la esfera a otra de idéntica para medir mediante un instrumento, similar a la balanza de torsión de Coulomb, la fuerza de repulsión y separación entre ambas. Se obtenía así una estimación de la carga en unidades electroestáticas. En la segunda parte, se descargaba la botella con la esfera, a través de un circuito de cobre en forma de círculo, para medir la fuerza que ejercía sobre una aguja magnetizada en su centro. Se obtenía así una estimación de la misma carga en unidades electrodinámicas. Weber sospechaba que el ratio entre ambas mediciones guardaba alguna relación con la velocidad de la luz. Sin embargo, la hipótesis del doble fluido había introducido un factor 2 en las ecuaciones que no le permitió una interpretación en que fenómenos ópticos y electromagnéticos se unificaran por completo.

Maxwell identificó correctamente que el factor 2 introducido en la estimación del ratio era debido a que se consideraban cargas opuestas circulando en ambas direcciones al mismo tiempo. Si se aplicaba la hipótesis del fluido único y se consideraba que toda la carga se desplazaba en una sola dirección se
Figura 10. Aparato como el utilizado por Weber y Kohlrausch para medir la fuerza magnética

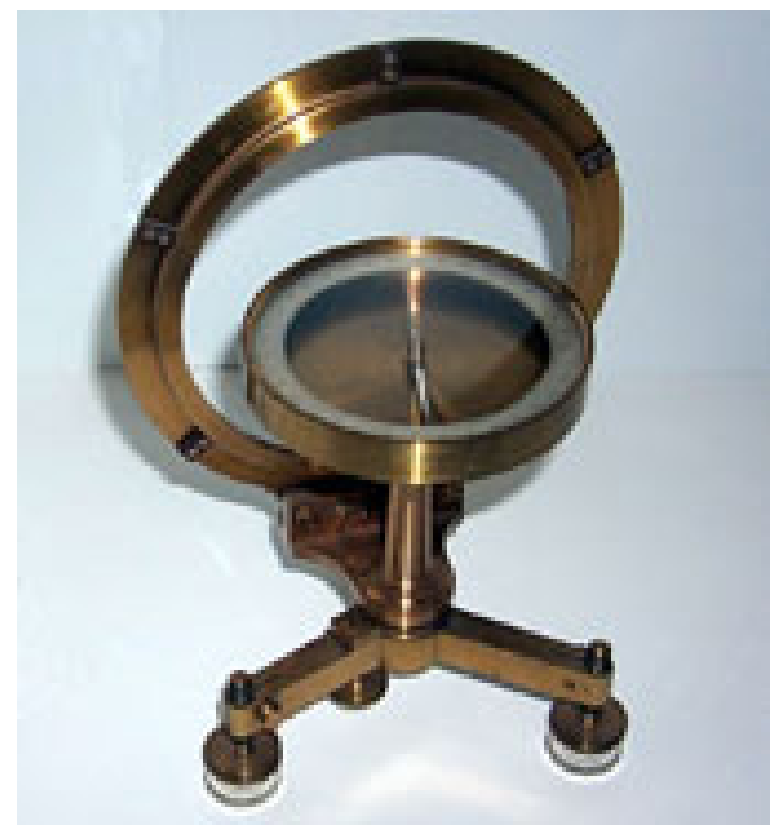

obtenía un valor de $310.740 .000 .000 \mathrm{~mm} / \mathrm{s}$. Como acababa de demostrar, este valor coincidía con la velocidad de propagación de las ondas transversales en el medio electromagnético. Por otro lado, Hippolyte Fizeau había realizado un experimento sin utilizar ningún aparato eléctrico o magnético en que estimaba la velocidad de propagación de la luz en $314.858 .000 .000 \mathrm{~mm} / \mathrm{s}$. Maxwell concluyó que esta coincidencia tenía que implicar una naturaleza electromagnética de la luz: "...difícilmente podemos evitar la inferencia de que la luz consiste en ondulaciones transversales del mismo medio que es la causa de los fenómenos eléctricos y magnéticos.".

Por último, en la Parte 4 (Maxwell, 1861b), titula$\mathrm{da}$ "The Theory of Molecular Vortices applied to the Action of Magnetism on Polarized Light", usaba el modelo para explicar uno de los fenómenos que le habían inspirado para idear el modelo de los vórtices o células rotantes. El efecto descubierto por Faraday unos quince años atrás consistía en la rotación del plano de polarización de una onda lumínica en la dirección de su propagación por el efecto de un campo magnético. Con el estudio del efecto sobre la luz polarizada de la rotación de los vórtices y el movimiento de las partículas eléctricas afianzaba el resultado más importante de este artículo: la luz es una onda electromagnética. 


\section{LAS ECUACIONES DE MAXWELL}

Que había algún tipo de relación entre los fenómenos eléctricos y ópticos era algo que se sospechaba desde hacía mucho tiempo. Era evidente que las descargas eléctricas producían luz pero esto podía ser explicado sin necesidad de identificar ambos fenómenos. De la teoría de la visión también parecía desprenderse algún tipo de relación entre la luz percibida por el ojo y las señales emitidas por los nervios presumiblemente eléctricos. Sin embargo, el artículo "On Physical Lines of Force" (Maxwell, 1861a, 1861b, 1862a, 1862b), construía una teoría solida que relacionaba definitivamente electromagnetismo y óptica. La luz era una onda electromagnética.

No contento con eso, Maxwell siguió desarrollando su teoría del campo electromagnético. Volvió a abordar el tema utilizando una aproximación cercana a la mecánica analítica de Lagrange. Este había hecho un gran esfuerzo para reducir la mecánica de Newton a un conjunto de formulas generales de sencilla aplicación. Había deducido las leyes de la mecánica utilizando simplemente el cálculo diferencial y el principio de mí- nima acción, sin apoyar sus deducciones en la geometría. Había expresado las energías cinética y potencial de un sistema material determinando la configuración de sus partes por tantas variables como grados de libertad. A partir de ellas se podían obtener por derivación las ecuaciones del movimiento.

Aplicando este enfoque al fenómeno electromagnético, Maxwell realizó un nuevo trabajo que fue leído ante la Royal Society en diciembre de 1864 . Al cabo de unos meses fue publicado en sus Transactions como un artículo de siete partes bajo el nombre "A Dynamical Theory of the Electromagnetic Field" (Maxwell, 1865). En la Parte III titulada "General Equations of the Electromagnetic Field" se presentaba un sistema de ocho expresiones. Eran dos ecuaciones escalares y seis vectoriales formadas por tres ecuaciones para cada eje del espacio. Se trataba pues en total de un sistema de veinte ecuaciones con veinte variables. Algunas de estas ecuaciones ya habían ido apareciendo en sus artículos anteriores pero aquí estaban agrupadas por primera vez bajo el nombre ecuaciones generales del campo electromagnético (Simpson, 1997, 2010; Gabàs, 2012).

Figura 11. Forma moderna de las ecuaciones de Maxwell para el campo electromagnético

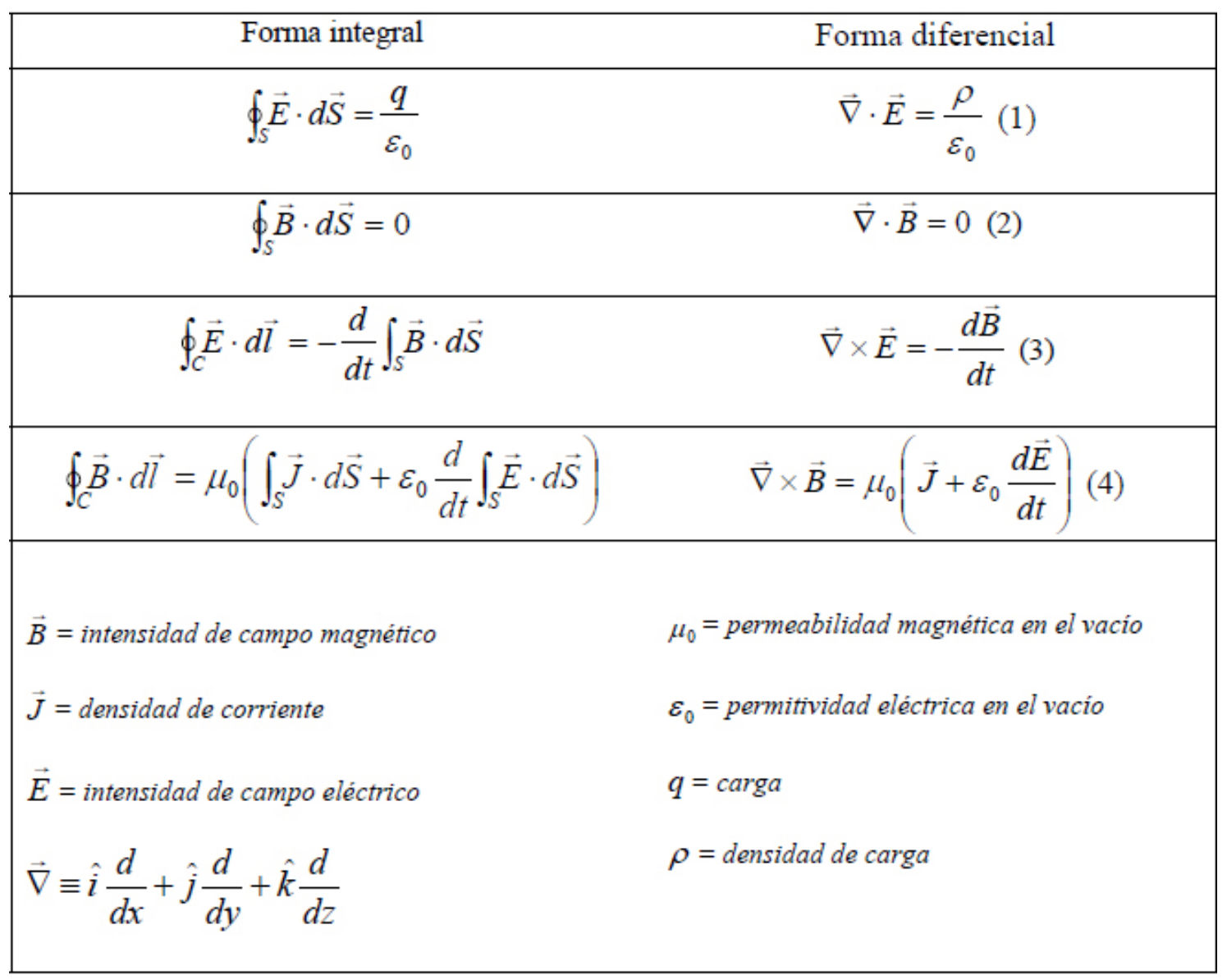


Después de pasar por numerosos refinamientos sucesivos, actualmente se conoce como las ecuaciones de Maxwell para el campo electromagnético a un conjunto de cuatro ecuaciones vectoriales (Fleisch, 2008). La primera es conocida como ley de Gauss para el campo eléctrico (1). Según ella, el flujo eléctrico a través de una superficie cerrada es proporcional a la suma de la carga total que alberga en su interior. El flujo eléctrico es análogo al de la mecánica de fluidos. Se utiliza para describir el campo eléctrico en relación a una superficie, es decir, cuál es la componente del campo perpendicular a dicha superficie. Aunque en la formulación original (Maxwell, 1865) no aparecía en su forma actual, puede obtenerse fácilmente combinando la ecuación de la electricidad libre (G) con la ecuación de la elasticidad eléctrica (E). En la formulación original aparecía la elasticidad eléctrica en lugar de su inversa la permitividad.

El operador nabla es la derivada parcial de cada componente del vector en cada una de las direcciones del espacio. Seguido de un producto escalar (·) representa la divergencia o la magnitud de ganancia o pérdida de intensidad de un campo vectorial en un punto. Seguido de un producto vectorial $(x)$ representa el rotacional o magnitud de la inducción a rotación de un campo vectorial alrededor de un punto. Los términos rotacional o curl y convergencia fueron sugeridos por el propio Maxwell para referirse a los distintos tipos de variación en un campo de vectores. Actualmente se siguen utilizando salvo que el término convergencia ha sido sustituido por su negativo, divergencia o div.

La segunda de las ecuaciones es conocida como ley de Gauss para el campo magnético (2). Según ella el flujo magnético a través de cualquier superficie cerrada es siempre nulo. Esto equivale a decir que todas las líneas de fuerza magnética que entran en tales superficies salen de igual manera, o lo que es lo mismo es imposible encontrar en su interior una fuente o sumidero de campo. Esto implica la no existencia de los polos magnéticos aislados y que las líneas de fuerza de los campos magnéticos deben ser cerradas. Esta expresión no aparecía en la formulación original (Maxwell, 1865). Fue introducida al conjunto en trabajos de autores posteriores como Heinrich Hertz para dar mayor simetría al conjunto.

La tercera de las ecuaciones es conocida como ley de Faraday (3). Según ella la circulación de la intensidad de campo eléctrico en un circuito cerrado (fuerza electromotriz) es directamente proporcional a la rapidez con que varia el flujo magnético que atraviesa una superficie cualquiera con dicho circuito como borde Esto implica que un campo magnético variable en el tiempo provoca la existencia de un campo eléctrico. Aunque en la formulación original (Maxwell, 1865) no aparecía en su forma actual, puede obtenerse fácilmente a partir de las ecuaciones de las fuerzas magnéticas (B) sustituyendo el momento electromagnético por la intensidad de campo eléctrico. Maxwell había utilizado el momento electromagnético para describir el estado electro-tónico de Faraday (Maxwell, 1864). La variación temporal del momento equivale a la intensidad de campo eléctrico. Este cambio en la formulación fue introducido por Oliver Heaviside intentando buscar una cierta simetría entre campo eléctrico y magnético. Heaviside también aplicaría la notación vectorial utilizando una flechita encima de las letras en mayúsculas para referirse a vectores.

La cuarta y última de estas ecuaciones es conocida como ley de Ampère-Maxwell (4). Ampère había encontrado una relación para un campo magnético y una corriente eléctrica que no variaban en el tiempo. Según la expresión actual de esta ley la corriente eléctrica que atraviesa una superficie abierta finita es proporcional a la circulación de la intensidad de campo magnético en la curva cerrada que constituye el borde de dicha superficie. Cuando se considera esta ley a lo largo de un circuito eléctrico se puede ver que en la parte donde está la diferencia de potencial hay una discontinuidad. Maxwell introdujo un nuevo término en que se consideraba en ese punto la variación temporal del flujo eléctrico como constituyente de unas pequeñas corrientes de desplazamiento. Este disminuía con la fuerza electromotriz a la vez que la carga se equilibraba entre los extremos por efecto de la corriente. La ley de Ampère-Maxwell (4) puede obtenerse a partir de la formulación original (Maxwell, 1865) fusionando las ecuaciones de la corriente eléctrica (C) y las ecuaciones de la corriente total (A). Una forma fusionada de ambas expresiones ya había aparecido en el anterior artículo de Maxwell (Maxwell, 1862a). Pero allí, la variación del desplazamiento eléctrico con el tiempo aparecía en el otro lado de las ecuaciones, por necesidades en el desarrollo matemático de lo que allí trataba.

Finalmente, si consideramos estas ecuaciones en el vacio, la densidad de carga de la ecuación (1) y la densidad de corriente de la ecuación (4) son cero. Gracias al término de corrientes de desplazamiento las ecuaciones adquieren una gran simetría: 


$$
\begin{gathered}
\vec{\nabla} \cdot \vec{E}=0 \\
\vec{\nabla} \cdot \vec{B}=0 \\
\vec{\nabla} \times \vec{E}=-\frac{d \vec{B}}{d t} \\
\vec{\nabla} \times \vec{B}=\mu_{0} \varepsilon_{0} \frac{d \vec{E}}{d t}
\end{gathered}
$$

Una solución para este sistema consiste en dos planos de ondas transversales perpendiculares entre sí del campo eléctrico y magnético. Ambas están en fase y sus frentes se propagan a la velocidad de la luz:

$$
c=\frac{1}{\sqrt{\mu_{0} \varepsilon_{0}}}
$$

\section{Consolidación de la teoría de las ondas electromagnéticas}

Uno de los personajes que influyó más en la consolidación de la teoría de las ondas electromagnéticas fue Heinrich Hertz (Wiederkehr, 2007a, 2007b). Después de estudiar ingeniería en Dresde, se desplazó a Berlín para aprender física con Gustav Kirchoff y Hermann von Helmholtz. Tras obtener su doctorado, pasó un tiempo como asistente de Helmholtz. Este último, gran admirador de Weber, llamó su atención sobre la teoría electromagnética de Maxwell. Tras un periodo de profesor en la Universidad de Kiel, Hertz se trasla- dó en 1885 a la Universidad de Karlsruhe. Allí, siguiendo con sus trabajos, comprobó experimentalmente la existencia de las ondas electromagnéticas predichas por Maxwell. A tal efecto, construyó él mismo en el laboratorio de física de la Escuela Técnica Superior un emisor y un receptor.

Como emisor utilizó un aparato consistente en un circuito por donde pasaba corriente en el que había una discontinuidad. En cada uno de los extremos de la discontinuidad estaba situada una pequeña esfera de latón. Ambas estaban separadas por un pequeño espació por donde se producían descargas eléctricas en forma de chispas. Gracias a esta técnica pudo obtener unos campos eléctricos que variaban con una frecuencia suficientemente alta para ser detectados por un aparato receptor. Como receptor utilizó un artilugio formado por un cable cerrado en un punto de cuyo recorrido había también dos pequeñas esferas separadas por un pequeño espacio. Cuando las chispas saltaban en el emisor, las ondas electromagnéticas provocadas por estas descargas inducían una corriente en el receptor que provocaba también chispas en este.

Desafortunadamente la carrera de Hertz fue muy breve ya que murió de septicemia con treinta y seis años. Sin embargo, a partir de sus sencillas experiencias con las ondas electromagnéticas se desarrollaron todas las telecomunicaciones inalámbricas del siglo $\mathrm{XX}^{5}$.

Figura 12. Aparatos como los utilizados por Hertz en su experimento
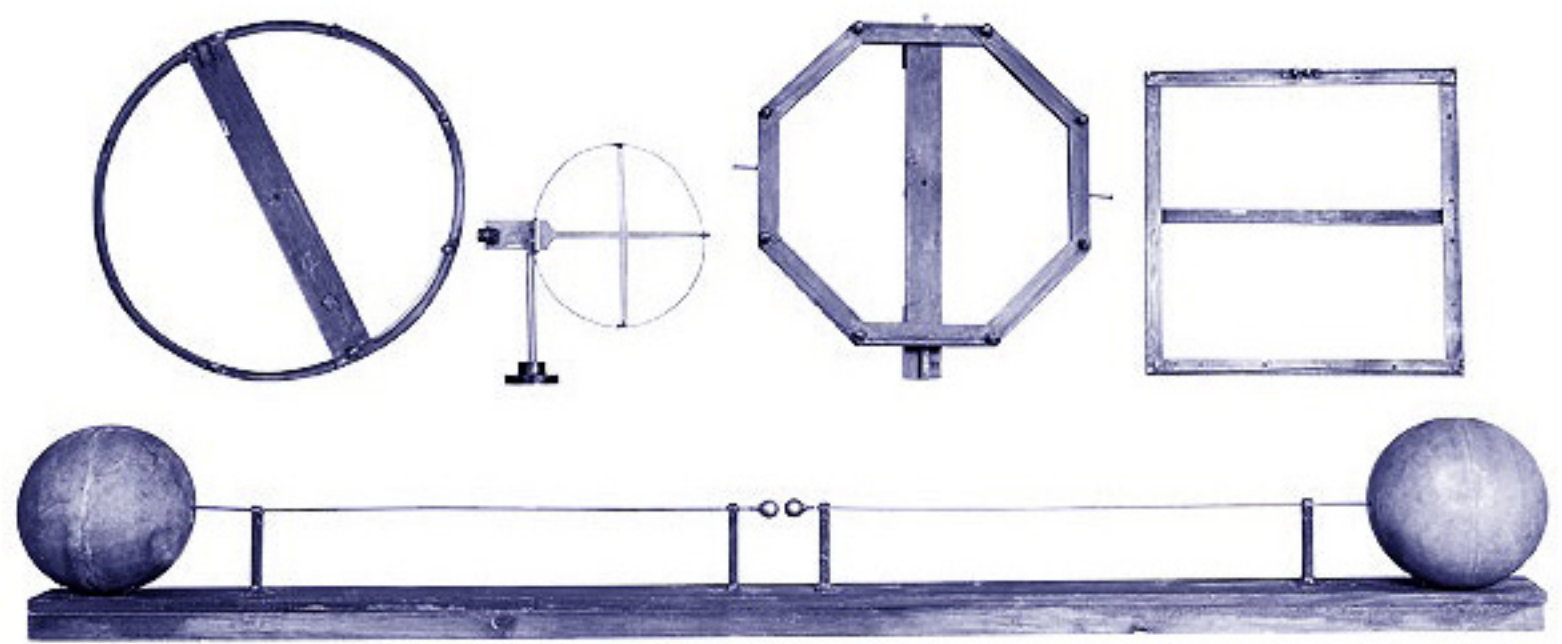


\section{NOTAS}

1 Maxwell leyó su artículo “On Faraday's lines of force" el 10 de diciembre de 1855 y el 11 de febrero de 1856, pero no aparece publicado en las actas de la Cambridge Philosophical Society hasta 1864
2 Maxwell la llamaba fuerza electromotriz cuyo significado actual es más restringido.

3 Maxwell la llamaba electricidad libre.
4 Esta es la causa del factor 2 que se mantiene actualmente en la definición del amperio.

5 La unidad de frecuencia se llama hercio en honor de Heinrich Hertz.

\section{BIBLIOGRAFÍA}

Assis, A. K. T., Reich, K. y Wiederkehr, K. H. (2002). Gauss and Weber's Creation of the Absolute System of Units in Physics. 21st Century Science and Technology, 15, 3, pp. 40-48.

Assis, A. K. T. (2003). On the first electromagnetic measurement of the velocity of light by Wilhelm Weber and Rudolf Kohlrausch. En: Bevilacqua F. y Giannetto E. A. (eds.) Volta and the History of Electricity. Milano: Università degli Studi di Pavia e Editore Ulrico Hoepli, pp. 267-286.

Campbell, L. y Garnett, W. (1884). The life of James Clerk Maxwell. London: Macmillan \& Co.

Crowther, J. C. (1945). J. Prescott Joule, WiIliam Thompson, J. Clerk Maxwell (hombres de ciencia británicos del siglo XIX). Buenos Aires, México: Espasa - Calpe Argentina.

Díaz-Hellín, J. A. (2001). El gran cambio de la Física. Faraday. Madrid: Nivola.

Elbel, M. (1993). Das Kohlrausch/WeberExperiment. Wege in der Physikdidaktik, Band 3, Erlangen: Palm \& Enke, pp. 37-42.

Fleisch, D. (2008). A student's guide to Maxwell's equations. Cambridge: Cambridge University Press. http://dx.doi. org/10.1017/CBO9780511984624

Gabàs, J. (2012). La naturaleza de la luz. Maxwell. Madrid: Nivola.

Glazebrook, R. T. (1896). James Clerk Maxwell and modern physics. New York: MacMillan \& Co. http://dx.doi. org/10.5962/bhl.title.32491

Hoffmann, B. (1983). Relativity and its roots. New York: Scientific American Books.

Kohlrausch, R. y Weber, W. (1857). Elektrodynamische Maassbestimmungen insbesondere Zurückführung der Stromintensitäts-Messungen auf mechanisches Maass. Abhandlungen der Kgl. Sächs. Gesellschaft der Wissenschaften. Mathe matisch-Physische Klasse, V, pp. 219-292.

Ladera, C. L. y Chela, J. (1979). Maxwell y Einstein. Ensayos biográficos. Caracas: Ediciones de la C. A. La Electricidad de Caracas y C. A. Luz Eléctrica de Venezuela.

Mahon, B. (2003). The man who changed everything. The life of James Clerk Maxwell. Chichester: Wiley.

Maxwell, J. C. (1849). On the theory of rolling curves. Transactions of the Royal Society of Edinburgh, XVI, pp. 519-540. http://dx.doi.org/10.1017/ S008045680002247X

Maxwell, J. C. (1851). On the descriptions of oval curves and those having a plurality of foci. Proceedings of the Royal Society of Edinburgh, II, pp. 89-91.

Maxwell, J. C. (1853). On the equilibrium of elastic solids. Transactions of the Royal Society of Edinburgh, XX, pp. 87-120.

Maxwell, J. C. (1855). Experiments on colour as perceived by the eye with remarks on colour blindness Transactions of the Royal Society of Edinburgh, XX, pp. 275-298.

Maxwell, J. C. (1859). On the Stability of the Motion of Saturn's Rings. London: Macmillan \& Co.

Maxwell, J. C. (1860a). Illustrations of the dynamical theory of gases. Part 1.On the motions and collisions of perfectly elastic spheres. The London, Edinburg and Dublin Philosophical Magazine and Journal of Science, XIX, pp. 19-32.

Maxwell, J. C. (1860b). Illustrations of the dynamical theory of gases. Part 2. On the process of diffusion of two or more kinds of moving particles among one another. The London, Edinburg and Dublin Philosophical Magazine and Journal of Science, XX, pp. 21-37.

Maxwell, J. C. (1861a). On physical lines of force. Part 1. The theory of molecular vortices applied to magnetic phenomena. The London, Edinburg and Dublin Philosophical Magazine and Journal of Science, XXI, pp. 161-175.
Maxwell, J. C. (1861b). On physical lines of force. Part 2. The theory of electrical vortices applied to electric currents. The London, Edinburg and Dublin Philosophical Magazine and Journal of Science, XXI, pp. 281-291, 338-348.

Maxwell, J. C. (1862a). On physical lines of force. Part 3. The theory of electrical vortices applied to statical electricity. The London, Edinburg and Dublin Philosophical Magazine and Journal of Science, XXIII, pp. 12-24.

Maxwell, J. C. (1862b). On physical lines of force. Part 4. The theory of electrical vortices applied to the action of magnetism on polarized light. The London, Edinburg and Dublin Philosophical Magazine and Journal of Science, XXIII, pp. 85-95.

Maxwell, J. C. (1864). On Faraday's lines of force, Transactions of the Cambridge Philosophical Society, X, pp. 27-83.

Maxwell, J. C. (1865). A dynamical theory of the electromagnetic field. Philosophical Transactions of the Royal Society of London, 155, pp. 459-512. http://dx.doi. org/10.1098/rstl.1865.0008

Maxwell, J. C. (1873). A Treatise on Electricity and Magnetism. London: Macmillan \& Co.

Maxwell, J. C. (1891/1954). A Treatise on Electricity and Magnetism. New York: Dover.

McDonald, D. K. C. (1966). Tres pilares de la ciencia moderna. Faraday, Maxwell y Kelvin. Buenos Aires: Editorial Universitaria de Buenos Aires.

Niven, W. D. (1890). The scientific papers of James Clerk Maxwell. Cambridge: Cambridge University Press.

Pérez, M. A. y Varela, P. (2003). Orígenes del electromagnetismo. Oersted y Ampère. Madrid: Nivola.

Simpson, T. K. (1997). Maxwell on the electromagnetic field. A guided study. New Brunswick - New Jersey - London: Rutgers University Press. 
Simpson, T. K. (2010). Maxwell's mathematical rhetoric: Rethinking the treatise on electricity and magnetism. Santa Fe: Green Lion Press.

Steward, I. (2008). Historia de las matemáticas en los últimos 10.000 años. Barcelona: Crítica.
Summers, J. (2002). Electricidad, periodismo y política. Franklin. Madrid: Nivola.

Wiederkehr K. H. (2007a). Heinrich Hertz (1857-1894) - Leben und Werk. En: Wolfschmidt G. (ed.) Von Hertz zum Handy - Entwicklung der Kommunikation. Hamburg: Nuncius Hamburgensis, pp. 61-68.
Wiederkehr K. H. (2007b). Die Karlsruher Experimente $(1886 / 1888)$ von Heinrich Hertz. En: Wolfschmidt G. (ed.) Von Hertz zum Handy - Entwicklung der Kommunikation. Hamburg: Nuncius Hamburgensis, pp. 69-76. 